UP-TP 94/1

\title{
TIME-LIKE SHOCK HADRONIZATION OF A SUPERCOOLED QUARK-GLUON PLASMA
}

\author{
M.I. Gorenstein, 1H.G. Miller, R.M. Quick and R.A. Ritchie
}

Department of Physics, University of Pretoria, Pretoria 0002, South Africa

\begin{abstract}
We study the energy-momentum and baryonic number conservation laws for quark-gluon plasma discontinuity transitions into hadron matter states. We find that the time-like shock hadronization of a supercooled quark-gluon plasma (when the normal vector to the discontinuity hypersurface is time- like) should take place. We consider some properties of this process, which is different from the standard space-like shock hadronization.
\end{abstract}

\footnotetext{
${ }^{1}$ Permanent address: Institute for Theoretical Physics, 252143 Kiev-143, Ukraine
} 
Relativistic shock phenomena have been widely discussed in recent years within their connection to high-energy heavy ion collisions (see, for example, [1]). Special attention has been paid to the phase transition between the hadronic matter (HM) and a quark-gluon plasma (QGP) expected to occur in such collisions. Different scenarios involving shocks as a possible mechanism for both the deconfinement transition and for QGP hadronization have been suggested. The only physical requirements which regulate the dynamics of these shock transitions are the energy-momentum and baryonic number conservation across the discontinuity front. The entropy growth condition (thermodynamical stability) and mechanical stability conditions [2] should be additionally checked to guarantee shock existence.

In the compressional shock model of heavy ion collisions the discontinuity in the thermodynamical parameters of strongly interacting matter appears due to the initial hydrodynamical (velocity) discontinuity in the system of colliding nuclei. The physical picture of (one-dimensional) compression shocks in heavy ion collisions is quite transparent (see, for example, [3]). It does not mean of course that the assumptions of this model are definitely realized in the real world - only experimental studies and theoretical analysis will clear up this question.

The status of the QGP shock-like hadronization, suggested in Ref.[4], is much less clear. Firstly, the origin of a hadronization discontinuity is quite different. It can result from smooth initial conditions in the course of the QGP expansion if a transition between HM and QGP is a 1-st order phase transition. In this case one can also expect the appearance of supercooled QGP states ahead of the discontinuity front and superheated HM states behind it. Secondly, the space-time properties of a discontinuity hypersurface as well as the physical meaning of the collective velocities have not yet been studied in this case. A recent consideration of shock-like QGP hadronization can be found in Refs.[5].

In our paper we analyze the discontinuity-like hadronization model. By admitting the existence of supercooled QGP and superheated HM we have the picture of discontinuity-like transitions which is essentially richer than only standard compression and rarefaction shocks (see also [6,7]). We find that discontinuity hadronization across the hypersurface with a time-like normal 4-vector (we call them t.l. shocks) should occur in the QGP dynamical evolution (i.e., in its expansion process). The mathematical properties of these t.l. shocks are in some respects rather similar to that of standard 
shock waves (we call them space-like shocks, or s.l. shocks), considered in Refs.[5], for which the normal 4-vector to shock front hypersurface is always space-like. Both kinds of shocks satisfy the same shock adiabat equation (Taub adiabat). The physical picture of t.l. schocks and its consequences for QGP hadronization are, however, quite different and will be studied in our paper.

The system evolution in relativistic hydrodynamics is governed by the energy-momentum tensor $T^{\mu \nu}=(\epsilon+p) u^{\mu} u^{\nu}-p g^{\mu \nu}$ and conserved charge currents (in our applications to heavy ion collisions it is the baryonic current $n u^{\mu}$ ). They consist of local thermodynamical fluid quantities (the energy density $\epsilon$, pressure $p$, baryonic density $n$ ) and the collective four-velocity $u^{\mu}=\left(1-\mathbf{v}^{2}\right)^{-1 / 2}(1, \mathbf{v})$. The continuous flows are the solutions of the hydrodynamical equations $\partial T^{\mu \nu} / \partial x^{\mu}=0, \partial n u^{\mu} / \partial x^{\mu}=0$ with specified initial and boundary conditions. These equations are nothing more than the differential form of the energy-momentum and baryonic number conservation laws. Along with these continuous flows, the conservation laws can also be realized in the form of discontinuous hydrodynamical flows which are called shock waves and satisfy the following equations:

$$
\begin{gathered}
T_{o}^{\mu \nu} \Lambda_{\nu}=T^{\mu \nu} \Lambda_{\nu}, \\
n_{o} u_{o}^{\mu} \Lambda_{\mu}=n u^{\mu} \Lambda_{\mu},
\end{gathered}
$$

where $\Lambda^{\mu}$ is the unit 4 -vector normal to the discontinuity hypersurface. In Eqs. $(1,2)$ the zero index corresponds to the initial state ahead of the shock front and quantities without an index are the final state values behind it. A general derivation of the shock equations (valid for both space-like and time-like normal vectors $\left.\Lambda^{\mu}\right)$ was given by L.Csernai [6].

The important constraint on the transitions $(1,2)$ (thermodynamical stability condition) is the requirement of non- decreasing entropy ( $s$ is the entropy density):

$$
s u^{\mu} \Lambda_{\mu} \geq s_{o} u_{o}^{\mu} \Lambda_{\mu} .
$$

To simplify our consideration and make our arguments more transparent we consider only one-dimensional hydrodynamical motion in what follows. To study the s.l. shock transitions one can always choose the Lorentz frame where the shock front is at rest. Then the hypersurface of shock discontinuity is $x_{\text {s.l.sh }}=$ const, and $\Lambda^{\mu}=(0,1)$. The shock equations $(1,2)$ in this 
(standard) case are:

$$
\begin{gathered}
T_{o}^{01}=T^{01}, \quad T_{o}^{11}=T^{11}, \\
n_{o} u_{o}^{1}=n u^{1} .
\end{gathered}
$$

Solving Eq.(4) one obtains

$$
v_{o}^{2}=\frac{\left(p-p_{o}\right)\left(\epsilon+p_{o}\right)}{\left(\epsilon-\epsilon_{o}\right)\left(\epsilon_{o}+p\right)}, \quad v^{2}=\frac{\left(p-p_{o}\right)\left(\epsilon_{o}+p\right)}{\left(\epsilon-\epsilon_{o}\right)\left(\epsilon+p_{o}\right)} .
$$

Substituting (6) into (5) we obtain the well known Taub adiabat equation (TA) [8]

$$
n^{2} X^{2}-n_{o}^{2} X_{o}^{2}-\left(p-p_{o}\right)\left(X+X_{o}\right)=0,
$$

in which $X \equiv(\epsilon+p) / n^{2}$, and it therefore contains only thermodynamical variables.

For discontinuities on a hypersurface with a time-like normal vector $\Lambda^{\mu}$ one can always choose another convenient Lorentz frame ("simultaneous system") where the hypersurface of the discontinuity is $t_{t . l . s h}=$ const and $\Lambda^{\nu}=(1,0)$. Equations $(1,2)$ are then

$$
\begin{gathered}
T_{o}^{00}=T^{00}, \quad T_{o}^{10}=T^{10}, \\
n_{o} u_{o}^{0}=n u^{0} .
\end{gathered}
$$

Solving Eq.(8) we find

$$
\tilde{v}_{o}^{2}=\frac{\left(\epsilon-\epsilon_{o}\right)\left(\epsilon_{o}+p\right)}{\left(p-p_{o}\right)\left(\epsilon+p_{o}\right)}, \quad \tilde{v}^{2}=\frac{\left(\epsilon-\epsilon_{o}\right)\left(\epsilon+p_{o}\right)}{\left(p-p_{o}\right)\left(\epsilon_{o}+p\right)},
$$

where we use " $\sim$ " sign to distinguish the t.l. shock case (10) from the standard s.l. shocks of (6). Substituting (10) into (9) one finds the equation for t.l. shocks which is identical to the TA of Eq.(7). We stress that the intermediate steps (Eqs.(10) and (6)) are, however, quite different. Note, that the two solutions, Eqs.(10) and (6), are connected to each other by simple relations

$$
\tilde{v}_{o}^{2}=\frac{1}{v_{o}^{2}}, \quad \tilde{v}^{2}=\frac{1}{v^{2}},
$$

between velocities for s.l. shocks and t.l. shocks. These relations show that only one kind of transition can be realized for a given initial state and final 
state. Physical regions $[0,1)$ for $v_{o}^{2}, v^{2}(6)$ and for $\tilde{v}_{o}^{2}, \tilde{v}^{2}(10)$ can be easily found in $(\epsilon-p)$-plane. For a given initial state $\left(\epsilon_{o}, p_{o}\right)$ they are shown in Fig.1. If one takes as initial and final states only states which are thermodynamically equilibrated it can be proven that the physical conditions $0 \leq v_{o}^{2}, v^{2} \leq 1$ are satisfied only for s.l. shocks for any EOS which gives the speed of sound in the medium smaller than or equal to 1 . The TA passes then through the point $\left(\epsilon_{o}, p_{o}\right)$ (which is called the centre of TA) and lies as a whole in the regions I and IV in Fig.1. The compressional s.l. shock transitions into region IV and rarefaction s.l. shock transitions into region I are called detonation and deflagration respectively. For supercooled initial QGP states the TA no longer passes through the point $\left(\epsilon_{o}, p_{o}\right)$ and new possibilities of t.l. shock hadronization $((8,9)$ shock transitions to regions III and VI in Fig. 1) appear.

To study any hydrodynamical problem quantitatively we need one more equation - an equation of state (EOS) which expresses local thermodynamical parameters in terms of two independent variables. The most convenient way is to present it in the form of $p=p(T, \mu)$ with temperature $T$ and baryonic chemical potential $\mu$ as independent variables. All other thermodynamical functions can be found then from the following thermodynamical identities:

$$
s=\left(\frac{\partial p}{\partial T}\right)_{\mu}, n=\left(\frac{\partial p}{\partial \mu}\right)_{T}, \epsilon=T s+\mu n-p .
$$

For HM we use a thermodynamically consistent "excluded volume" model [9] where the hadronic pressure can be expressed in terms of ideal gas pressures as $\left(V_{i}^{*}\right.$ is the proper volume for a hadron of type " $i$ "):

$$
p_{h}=\sum_{i} p_{i}^{i d}\left(T, \tilde{\mu}_{i}\right), \quad \tilde{\mu}_{i}=\mu_{i}-V_{i}^{*} p_{h} .
$$

The hadron pressure is suppressed compared to the ideal gas pressure because of the shift, $-V_{i}^{*} p_{h}$, in the particle chemical potentials. In the entropy, baryonic and energy density, as follows from Eqs.(12), the additional suppression factor $\left[1+\sum_{i} V_{i}^{*} n_{i}^{i d}\left(T, \tilde{\mu}_{i}\right)\right]^{-1}$ appears. For example, the HM entropy density is given by

$$
s_{h}=\frac{\sum_{i} s_{i}^{i d}\left(T, \tilde{\mu}_{i}\right)}{1+\sum_{i} V_{i}^{*} n_{i}^{i d}\left(T, \tilde{\mu}_{i}\right)} .
$$

For the QGP we use the "cut-off" model [10] which omits the lowmomentum contribution $\left(k \leq k_{c}\right)$ of the quark and gluon spectra. In contrast 
to the bag model EOS for the QGP, the cut-off model reproduces the lattice results reasonably well (see [11]). Besides, it has one more advantage in our problem. When we include supercooled states of the QGP we still have a positive QGP pressure whereas in the bag model we would encounter negative values of QGP pressure even for a small amount of supercooling.

In our consideration we have restricted ourselves to a pion-nucleon gas with the proper volumes chosen as $V_{\pi}^{*}=V_{N}^{*}=1.63 \mathrm{fm}^{3}$ (which gives a radius of $0.73 \mathrm{fm}[9]$ ) and have chosen $k_{c}=900 \mathrm{MeV}$ (the same constant value for both $u, d$-quarks and gluons) in order to get a reasonable $(T-\mu)$ phase diagram. The phase transition line obtained by equating the HM and QGP pressures is shown in Fig.2.

If we admit supercooled QGP states for the initial states in shock transitions and/or superheated HM states for the final states, the position of the TA in the $(\epsilon-p)$-plane changes drastically. It does not pass in this case through the initial (non-equilibrium) point $\left(\epsilon_{o}, p_{o}\right)$. The TA $(7)$ for our EOS and a supercooled initial QGP state lies in the following regions of the $(\epsilon-p)$ plane (see Fig.1): I (s.l. deflagration), II (unphysical region both for (6) and (10)), III (t.l. detonation) and IV (s.l. detonation). In Fig.3 we show several examples of TAs for the supercooled QGP states with $\mu_{o}=1000 \mathrm{MeV}$ and $T_{o}=127.5,124.0,122.25 \mathrm{MeV}$. Black points correspond to thermodynamically stable (entropy growth condition (3) is fulfilled) shock transitions to the final hadron states. In a s.l. deflagration from QGP states, one observes the release of the large latent heat which is transformed into the collective motion of the produced HM (see Ref. [4]). A s.l. detonation of supercooled QGP (considered in Refs. [5]) has quite different behaviour. The QGP has a large velocity and small density. On the other side of the shock front HM appears with lower velocity but larger density. The space-time position of a discontinuity hypersurface was not however considered in the previous studies of shock-like QGP hadronization. We stress that this hypersurface is most probably (see examples below) the hypersurface with time-like normal vector. It leads to a t.l. detonation of supercooled QGP. Final HM states in this case belong to the intermediate part of TA between s.l. deflagration and s.l. detonation (see Figs.1 and 3).

The simplest solution of the t.l. shock equations $(8,9)$ is

$$
\tilde{v}^{2}=\tilde{v}_{o}^{2}=0, \epsilon=\epsilon_{o}, n=n_{o}, \quad p \neq p_{o} .
$$

This is a time-like analog of the simplest s.l. shock solution $v^{2}=v_{o}^{2}=0, p=$ 
$p_{o}, \epsilon \neq \epsilon_{o}, n \neq n_{o}$ which is called a contact discontinuity. Relations (14) take place, for example, in the one-dimensional scaling expansion (Bjorken model [12]) $v=x / t$ where the normal vector to the hypersurface of constant proper time $\left(t^{2}-x^{2}\right)^{1 / 2}=$ const (in which the thermodynamical parameters of the expanding QGP are fixed) has the form $\Lambda^{\nu}=(1,0)$ in the rest frames of each fluid element. The minimal supercooling of the QGP to have a thermodynamically stable t.l. shock hadronization of this type is shown in Fig.2. Note that for $\mu=1000 \mathrm{MeV}$, the temperature for minimal supercooling is $125.78 \mathrm{MeV}$, so that the initial QGP state lies above the minimal supercooling line for TAs in Fig.3a and below in Figs.3b and 3c.

In the general case when $\tilde{v}_{o}^{2}$, defined by the hydrodynamical solution, is not zero, Eqs. $(7,10)$ give us all the quantities of the final state.

In conclusion we have studied energy-momentum and baryonic number conservation in shock-like hadronization of a supercooled QGP. In the expanding system the space-time hypersurface of "critical" (hadronization) QGP parameters is most probably a hypersurface with a time-like normal 4 - vector. This is the case for the Bjorken model in $1+1$ dimensions and also for all known hydrodynamical solutions in the central rapidity region. This means that the hadronization of a supercooled QGP arises as a t.l. shock which is different from the (standard) case of s.l. shock hadronization. Several examples of the TA have been analyzed in the $(\epsilon-p)$-plane (Fig.3) to show different physical regions for final hadron states. The simplest t.l. shock hadronization (14) takes place for the scaling expansion of the QGP. We have also found (see Fig.3) that solution (14) corresponds to the minimal supercooling for thermodynamically stable t.l. shock hadronization. This minimal supercooling, as it is seen from Fig.2, is not very strong especially at small baryonic density. For zero baryonic density the minimal supercooling temperature is only $6 \%$ smaller than that of the phase transition.

\section{Acknowledgements}

We acknowledge the financial support of the Foundation for Research Development, Pretoria. M.I.G. is indebted to L.P.Csernai for useful discussions of time-like shocks. He also gratefully acknowledges the warm hospitality at University of Pretoria, where this work has been done. 


\section{Figure Captions}

Fig.1. Possible final states in the (energy density-pressure)-plane for shock transitions from the initial state $\left(\epsilon_{o}, p_{o}\right)$. I and IV are the physical regions for s.l. shocks, III and VI for t.l. shocks. II and V are unphysical regions for both types of shocks. Note, that only states with $p \leq \epsilon$ are possible for any physical equation of state in relativistic theory.

Fig.2. The phase diagram of strongly interacting matter in $(\mu-T)$-plane. The solid line shows the phase transition between HM and QGP. The dashed line corresponds to the minimal supercooling of the QGP having thermodynamically stable t.l. shock hadronization (14) in the scaling expansion of the QGP.

Fig.3a-c. Taub adiabats for the supercooled QGP initial states with $\mu_{o}=$ $1000 \mathrm{MeV}$ and $T_{o}=127.5 \mathrm{MeV}(\mathrm{a}), T_{o}=124.0 \mathrm{MeV}(\mathrm{b}), T_{o}=122.25 \mathrm{MeV}$ (c).

Black points correspond to the thermodynamically stable (entropy growth condition (3) is fulfilled) shock transitions to the final hadron states. White points are thermodynamically unstable shock transitions. The TA in the unphysical region II (where both $v_{o}^{2}, v^{2}(6)$ and $\tilde{v}_{o}^{2}, \tilde{v}^{2}(10)$ are negative) are shown by dashed lines. The points marked by large circles on the boundary between regions III and IV correspond to the limiting (unphysical) case when

both t.l. shock velocities (6) and s.l. (10) in both phases are equal to 1 . The straight solid line is $p=\epsilon-\epsilon_{o}+p_{o}$. 


\section{References}

[1] R.Stock, Phys. Rep. 135 (1986) 259;

H.Stöcker and W.Greiner, Phys. Rep. 137 (1986) 277.

[2] M.I.Gorenstein and V.I.Zhdanov, Z. Phys. C34 (1987) 79;

K.A.Bugaev and M.I.Gorenstein, J. Phys. G13 (1987) 1231;

K.A.Bugaev, M.I.Gorenstein and V.I.Zhdanov, Z. Phys. C39 (1988) 365.

[3] K.A.Bugaev, M.I.Gorenstein, B.Kämpfer and V.I.Zhdanov, Phys. Rev. D40 (1989) 2903;

K.A.Bugaev, M.I.Gorenstein, D.H.Rischke, Phys. Lett. B255 (1991) 18.

[4] L.Van Hove, Z. Phys. C21 (1983) 93; C27 (1985) 15.

[5] N.Bilić, J.Cleymans, E.Suhonen and D.W. von Oertzen, Phys. Lett. B311 (1993) 266;

N.Bilić, J.Cleymans, K.Redlich and E.Suhonen, Preprint CERN-TH 6923 and BI-TP-93/32.

[6] L.Csernai, Zh. Eksp. Teor. Fiz. (Russ.) 92 (1987) 379; Sov. Phys. JETP 65 (1987) 216.

[7] A.K.Holme, E.F.Staubo, L.P.Csernai, E.Osnes and D.Strottman, Phys. Rev. D40 (1989) 3735.

[8] A.H.Taub, Phys. Rev. 74 (1948) 328.

[9] D.H.Rischke, M.I.Gorenstein, H.Stöcker and W.Greiner, Z. Phys. C51 (1991) 485;

J.Cleymans, M.I.Gorenstein, J.Stalnacke and E.Suhonen, Phys. Scripta 48 (1993) 277.

[10] F.Karsch, Z. Phys. C38 (1988) 147;

M.Gorenstein and O.Mogilevsky, Z. Phys. C38 (1988) 161;

D.H.Rischke, M.I.Gorenstein, H.Stöcker and W.Greienr, Phys. Lett. 237B (1990) 153;

J.Engels et al., Z. Phys. C42 (1989) 341, Phys. Lett. B252 (1990) 625. 
[11] D.H.Rischke et al., Phys. Lett. B278 (1992) 19; Z. Phys. C56 (1992) 325; M.I.Gorenstein, W.Greiner and St.Mrówczyński, Phys. Lett. B286 (1992) 365 .

[12] J.D.Bjorken, Phys. Rev. D27 (1983) 140. 
This figure "fig1-1.png" is available in "png" format from: http://arxiv.org/ps/hep-ph/9406282v1 
$\frac{\overline{0}}{\stackrel{0}{5}}$

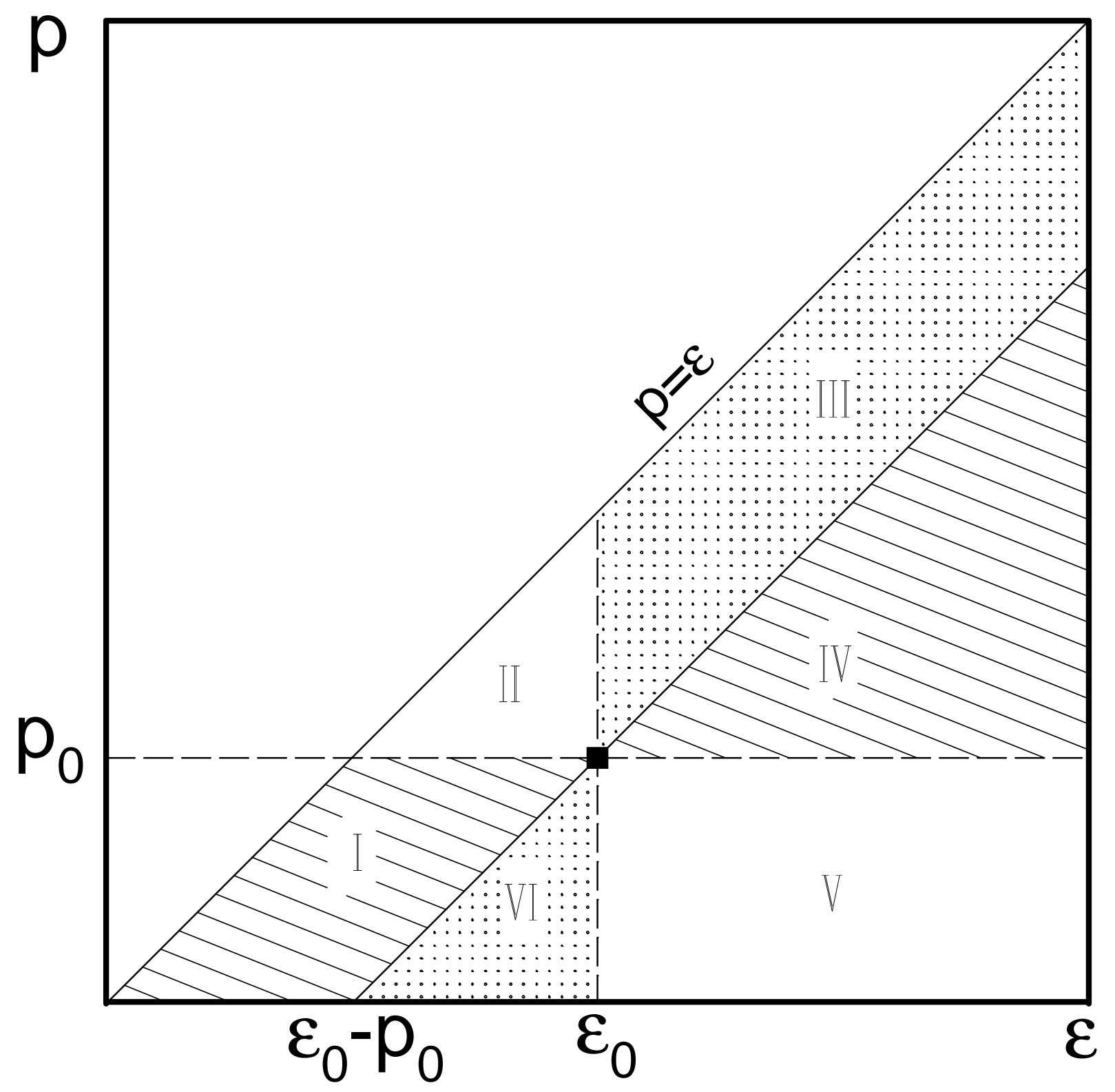


This figure "fig1-2.png" is available in "png" format from: http://arxiv.org/ps/hep-ph/9406282v1 
늠
믄

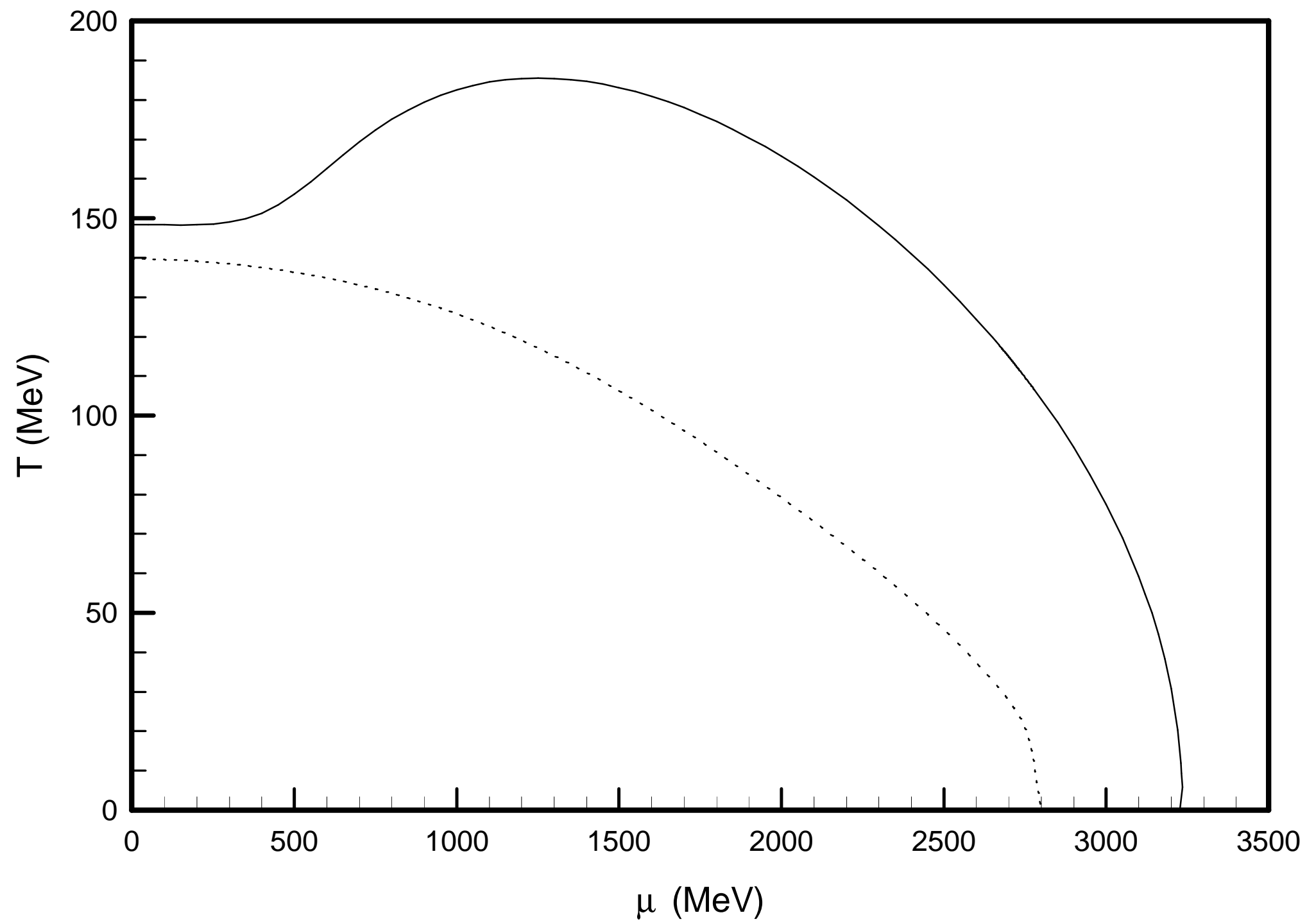


This figure "fig1-3.png" is available in "png" format from: http://arxiv.org/ps/hep-ph/9406282v1 


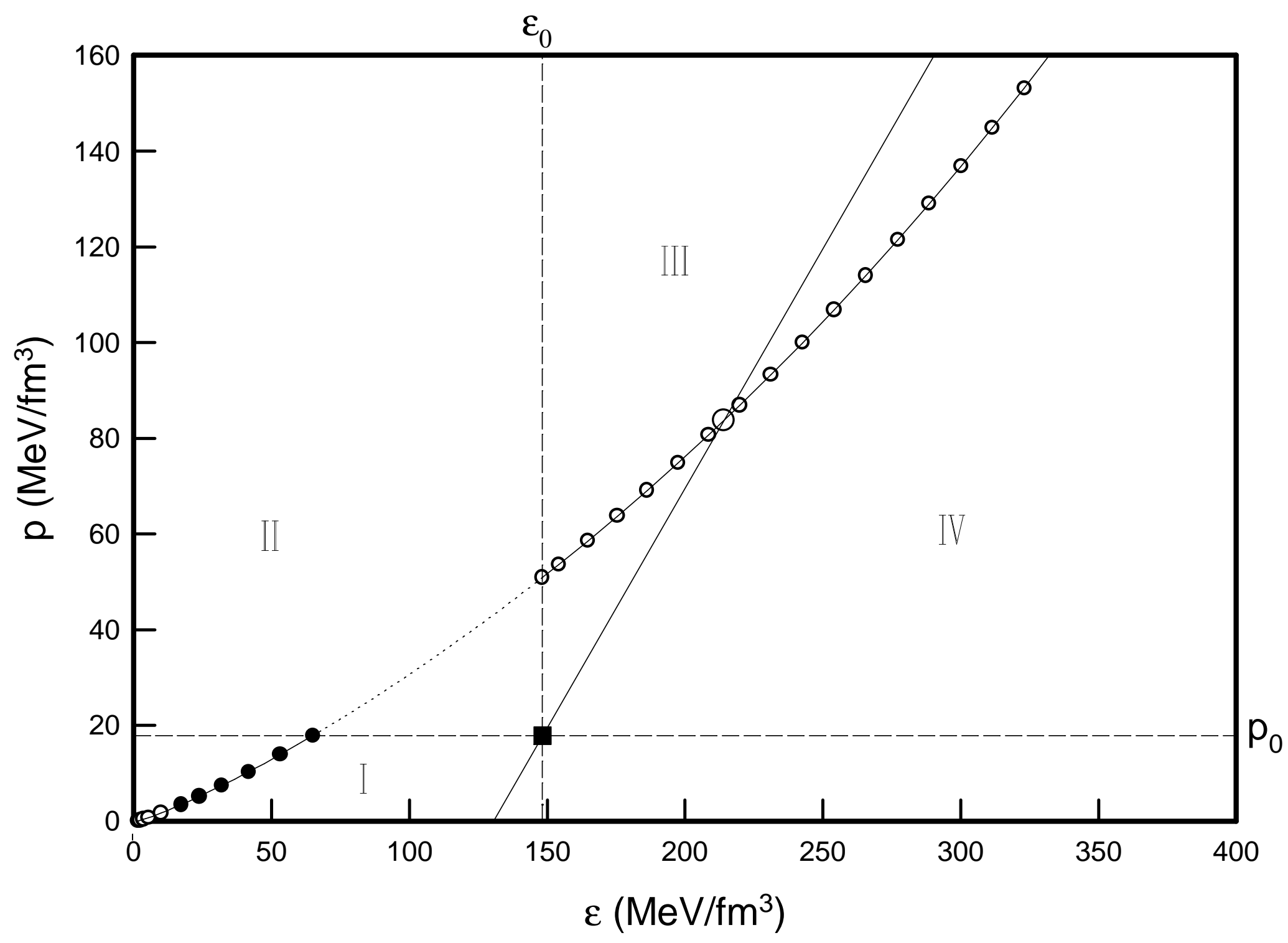


으
일
믄

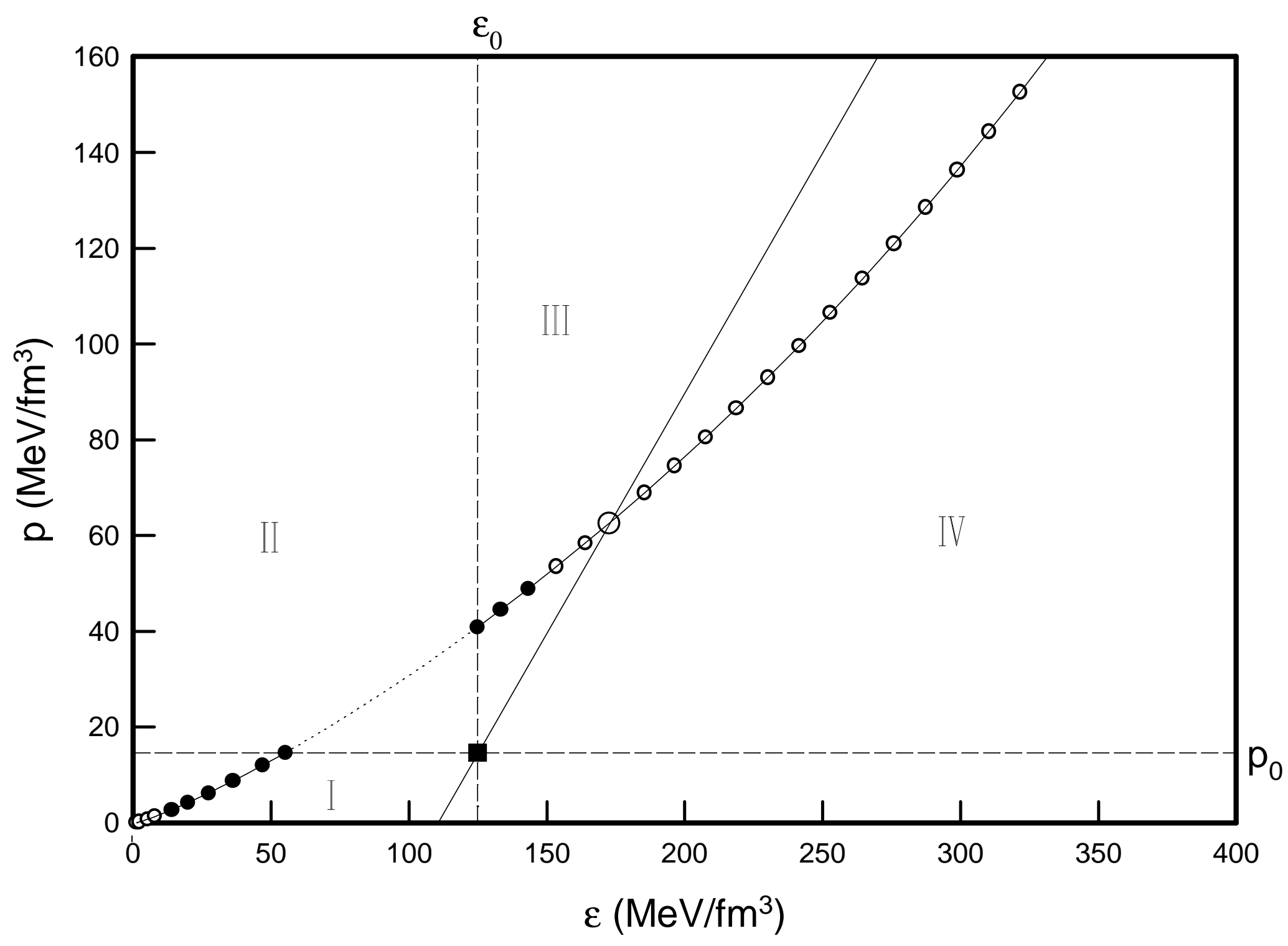


0
ल
ํㅣㄴ
믄

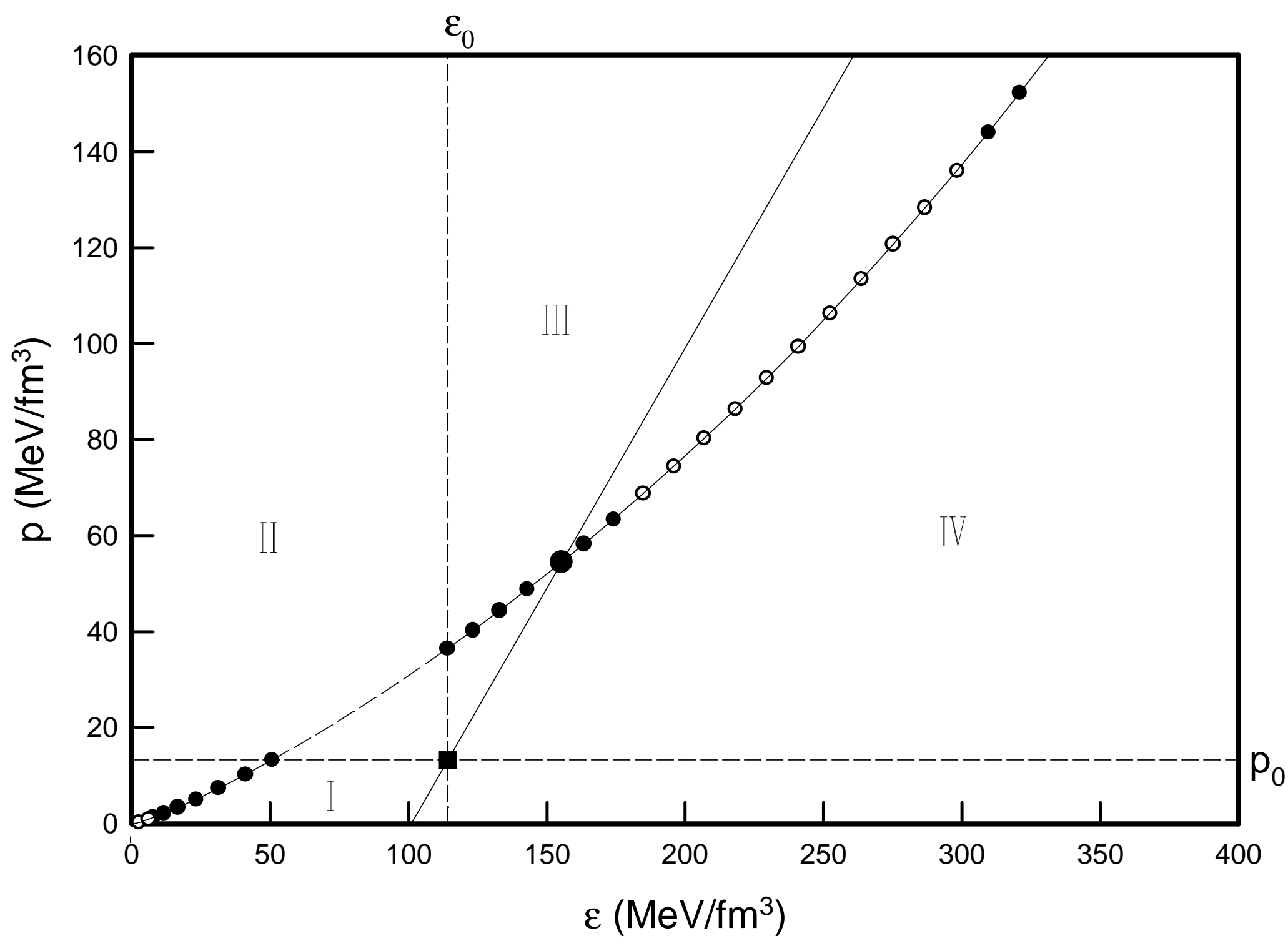


This figure "fig1-4.png" is available in "png" format from: http://arxiv.org/ps/hep-ph/9406282v1 
This figure "fig1-5.png" is available in "png" format from: http://arxiv.org/ps/hep-ph/9406282v1 